\title{
The importance of E-cadherin binding partners to evaluate the pathogenicity of E-cadherin missense mutations associated to HDGC
}

\author{
Joana Figueiredo $^{1,2}$, Ola Söderberg ${ }^{3}$, Joana Simões-Correia ${ }^{1,4}$, Karin Grannas ${ }^{3}$, Gianpaolo Suriano ${ }^{1}$ \\ and Raquel Seruca ${ }^{\star, 1,2}$
}

In hereditary diffuse gastric cancer (HDGC), $C D H 1$ germline gene alterations are causative events in $30 \%$ of the cases. In $20 \%$ of HDGC families, $C D H 1$ germline mutations are of the missense type and the mutation carriers constitute a problem in terms of genetic counseling and surveillance. To access the pathogenic relevance of missense mutations, we have previously developed an in vitro method to functionally characterize them. Pathogenic E-cadherin missense mutants fail to aggregate and become more invasive, in comparison with cells expressing the wild-type (WT) protein. Herein, our aim was to develop a complementary method to unravel the pathogenic significance of E-cadherin missense mutations. We used cells stably expressing WT E-cadherin and seven HDGC-associated mutations (five intracellular and two extracellular) and studied by proximity ligation assays (PLA) how these mutants bind to fundamental regulators of E-cadherin function and trafficking. We focused our attention on the interaction with: p120, $\beta$-catenin, PIPKI $\gamma$ and Hakai. We showed that cytoplasmic E-cadherin mutations affect the interaction of one or more binding partners, compromising the E-cadherin stability at the plasma membrane and likely affecting the adhesion complex competence. In the present work, we demonstrated that the study of the interplay between E-cadherin and its binding partners, using PLA, is an easy, rapid, quantitative and highly reproducible technique that can be applied in routine labs to verify the pathogenicity of E-cadherin missense mutants for HDGC diagnosis, especially those located in the intracellular domain of the protein.

European Journal of Human Genetics (2013) 21, 301-309; doi:10.1038/ejhg.2012.159; published online 1 August 2012

Keywords: HDGC; E-cadherin; CDH1 mutations; E-cadherin trafficking; E-cadherin binding partners; diagnostic method

\section{INTRODUCTION}

Hereditary diffuse gastric cancer (HDGC) is an autosomal dominant cancer syndrome characterized by a high risk of developing diffuse gastric cancer ${ }^{1-3}$ and lobular breast cancer ${ }^{4-6}$ during life-time. $\mathrm{CDH} 1$ germline gene alterations (mutations or deletions), resulting in E-cadherin inactivation, are the only causative events described till now and were identified in approximately $30 \%$ of HDGC cases. ${ }^{2,3,7}$ To date, 122 different germline mutations have been described in these families, ${ }^{8}$ being the majority of them of the nonsense type, leading to alternative premature termination codons. ${ }^{3}$ This type of $\mathrm{CDH} 1$ mutant transcripts is commonly downregulated by nonsensemediated decay leading to E-cadherin loss of function ${ }^{9}$ and these patients are considered high-risk carriers and are counseled to perform prophylactic total gastrectomy. ${ }^{10}$ In about $20 \%$ of HDGC families, carriers show $\mathrm{CDH} 1$ germline missense mutations ${ }^{11}$ and, in contrast to truncating mutations, their pathogenic significance is not straightforward, therefore constituting a problem in terms of genetic counseling and surveillance.

In 2004, Fitzgerald and Caldas ${ }^{10}$ suggested that the significance of CDH1 missense mutations should be assessed in at least four affected members within a HDGC family in combination with functional and transcript analysis to look for activation of cryptic splice sites. However, in most cases, this type of analysis is not possible to accomplish owing to the lack of biological material or to the small size of the families. To circumvent this limitation and improve genetic counseling, we developed functional in vitro assays and an in silico working model to characterize HDGC-associated E-cadherin germline missense mutations. ${ }^{12,13}$ This has allowed genetic counselors to assess the pathogenic significance of this type of $\mathrm{CDH1}$ mutations and survey these mutation carriers.

$\mathrm{CDH} 1$ gene encodes for E-cadherin, which is the most important protein for the establishment and maintenance of epithelial cell-cell adhesion and for the suppression of tumor invasion. ${ }^{14}$ Using functional in vitro assays, we demonstrated that cells expressing pathogenic $\mathrm{CDH} 1$ missense mutations fail to aggregate and become more invasive, in comparison with cells expressing wild-type (WT) E-cadherin, 5,12,15-22 supporting their pathogenic relevance.

Importantly, our group identified two $C D H 1$ germline missense mutations, R749W and E757K, which produce low total and surface E-cadherin expression, despite the normal RNA levels, due to endoplasmic reticulum-associated degradation (ERAD), a mechanism of protein quality control. ${ }^{21}$ These mutants raised a special interest

${ }^{1}$ IPATIMUP, Institute of Molecular Pathology and Immunology of the University of Porto, Porto, Portugal; ${ }^{2}$ Medical Faculty of the University of Porto, Porto, Portugal; ${ }^{3}$ Department of Immunology, Genetics and Pathology, Uppsala University, Uppsala, Sweden; ${ }^{4}$ Centre of Ophthalmology and Vision Sciences - IBILI, Coimbra, Portugal

*Correspondence: Dr R Seruca, IPATIMUP, Institute of Molecular Pathology and Immunology of the University of Porto, Rua Dr Roberto Frias, s/n, $4200-465$ Porto, Portugal. Tel: +351 22 5570700; Fax: +351 22 5570799; E-mail: rseruca@ipatimup.pt

Received 16 March 2012; revised 15 June 2012; accepted 19 June 2012; published online 1 August 2012 
because they affect the juxtamembrane domain of E-cadherin, reported as crucial for E-cadherin traffic dynamics. ${ }^{23,24}$

E-cadherin expression and stability at the cell surface of epithelial cells are tightly regulated by post-translational mechanisms, including exocytosis and endocytosis. ${ }^{23-25}$ The exocytic and endocytic trafficking orchestrate E-cadherin transport to adherens junctions, internalization, recycling, sequestration and degradation. ${ }^{23-26}$ The cytoplasmic domain of E-cadherin is the main actor in these processes. Newly synthesized E-cadherin is transported from the Golgi apparatus to the plasma membrane (PM), after the association of $\beta$-catenin and Type $\mathrm{I} \gamma$ phosphatidylinositol phosphate kinase (PIPKI $\gamma$ ) to the cytoplasmic tail of E-cadherin. ${ }^{27,28}$ At the PM, p120-catenin binds to the cadherin juxtamembrane domain, stabilizing and preventing the entry of E-cadherin into degradative endocytic pathways. ${ }^{29-32}$ E-cadherin deprived of p120 is prone to interact with other proteins, such as clathrin adapter proteins and Hakai, promoting E-cadherin internalization. ${ }^{32,33}$ After internalization, E-cadherin can be recycled back to the PM or targeted for degradation. ${ }^{23}$

In the present work, we aim to determine whether E-cadherin cytoplasmic mutations associated to HDGC interfere with the binding of key partners for E-cadherin trafficking and, as consequence, lead to E-cadherin deregulation and loss of function. As a direct application of this study, we aimed to establish a complementary methodology to uncover the pathogenic significance of missense mutants found in a germline context of HDGC.

We selected a panel of seven HDGC missense mutations (five intracellular and two extracellular) that have been proven to be pathogenic, ${ }^{16,18,19,21,34-38}$ and studied the ability of the mutant proteins to interact with direct E-cadherin binding partners using proximity ligation assays (PLA), a new tool to detect in situ proteinprotein interactions. ${ }^{39,40}$ Fundamentally, PLA is a method where protein-recognition events are converted into detectable DNA molecules. In this method, a pair of proximity probes (consisting of antibodies to which an oligonucleotide has been conjugated) is used to target the proteins of interest. Close proximity of such probes allows rolling-circle amplification of a circular DNA molecule, which is then detected by hybridization of complementary fluorescent oligonucleotides. ${ }^{39,40}$

We focused our attention in fundamental regulators of E-cadherin function and trafficking, such as p120, $\beta$-catenin, PIPKI $\gamma$ and Hakai. In order to validate our findings, we characterized the E-cadherin mutants for subcellular localization and functionality.

Overall, we showed that cytoplasmic E-cadherin mutations affect the interaction of at least one binding partner, leading to a deregulated protein trafficking, and this can compromise its cellular function as invasion suppressor and cell-cell adhesion promoter. Therefore, the analysis of the interplay between E-cadherin and its binding partners could be an avenue to assess E-cadherin functionality, especially in the particular case of E-cadherin cytoplasmic mutations. Our results suggest that PLA should be included in routine analysis, in combination with invasion, aggregation and flow cytometry assays, to provide a more robust evaluation of E-cadherin pathogenicity for HDGC diagnosis.

\section{MATERIALS AND METHODS}

\section{Plasmids construction}

E-cadherin mutants T340A, A634V, R749W, E757K, E781D, P799R and V832M found in the HDGC context, ${ }^{16,18,19,21,34-38}$ were constructed by site-directed mutagenesis in the entry vector CDH1pENTR 221 (Clone ID: IOH46767, Invitrogen, Grand Island, NY, USA), following the protocol described by Wang and Wilkinson. ${ }^{41}$ By LR recombination reaction, the open reading frame was subcloned in the pEF6/Myc-His vector (Invitrogen). The Mock vector was constructed by restriction of the CDH1pEF6/Myc-His with BsrGI (Biolabs, Ipswich, MA, USA). All the clonings were verified by direct sequencing.

\section{Cell culture}

CHO (Chinese hamster ovary) cells (ATCC number: CCL-61, Barcelona, Spain) were transfected with the following vectors: empty vector (Mock), WT hE-cad, T340A, A634V, R749W, E757K, E781D, P799R and V832M, using Lipofectamine 2000 (Invitrogen, Carlsbad, CA, USA), according to the manufacture procedure. The transfected cells were selected by antibiotic resistance to blasticidin $(5 \mu \mathrm{g} / \mathrm{ml})$. Cells were grown at $37^{\circ} \mathrm{C}$ under $5 \% \mathrm{CO}_{2}$ humidified air, in $\alpha$-MEM $(+)$ medium (Gibco, Invitrogen, Grand Island, NY, USA) supplemented with $10 \%$ fetal bovine serum (HyClone, Perbio, Cramlington, UK), 1\% penicillin/ streptomycin (Gibco, Invitrogen) and blasticidin (Gibco, Invitrogen).

\section{Proximity ligation assay}

Cells were seeded on 6-well plates on top of glass coverslips and grown to at least $80 \%$ confluence. Fixation was performed in ice-cold methanol for $20 \mathrm{~min}$ for PLA E-cadherin/ $\beta$-catenin, PLA E-cadherin/p120 and PLA E-cadherin/ PIPKI $\gamma$, or in formaldehyde for $30 \mathrm{~min}$ for PLA E-cadherin/Hakai and subjected to PLA using Duolink Detection kit (Olink Bioscience, Uppsala, Sweden), according to the manufacturer's instructions for Duolink Blocking solution and Detection protocol. Briefly, slides were blocked, incubated with antibodies directed against E-cadherin cytoplasmic domain (from BD Biosciences, Erembodegem, Belgium, mouse, 1:100 or from Cell Signaling Technologies, Danvers, MA, USA, rabbit, 1:30), $\beta$-catenin (Sigma, Saint Louis, MO, USA, rabbit, 1:100), p120 (BD Biosciences, mouse, 1:30), PIPKI $\gamma$ (BD Biosciences, mouse, 1:30) and Hakai (Santa Cruz Biotechnology, Santa Cruz, CA, USA, goat, 1:50) and thereafter incubated with PLA probes, which are secondary antibodies (anti-mouse Minus, anti-rabbit Plus or anti-goat Plus) conjugated to unique oligonucleotides. Amplification template oligonucleotides were hybridized to pairs of PLA probe and circularized by ligation. The formed DNA circle was then amplified using rolling circle amplification into a bundle of single-stranded DNA anchored to one of the antibodies, and was detected by addition of complementary oligonucleotides labeled with $\mathrm{Cy} 3$ fluorophore. The coverslips were mounted on slides using Vectashield with DAPI (Vector Laboratories, Burlingame, CA, USA). Images were acquired on a Carl Zeiss Apotome Axiovert 200M Fluorescence Microscope (Carl Zeiss, Jena, Germany), using $\times 20$ and $\times 40$ objectives. Images were taken with an Axiocam HRm camera and processed with the Zeiss Axion Vision 4.8 software. The quantification of the dot-like PLA products was performed using BlobFinder V3.2. ${ }^{42}$

\section{Western blotting}

Cells were lysed in cold Catenin lysis buffer - 1\% Triton X-100 (Sigma), 1\% Nonidet P-40 (Sigma) in PBS - enriched with a protease inhibitor cocktail (Roche, Indianapolis, IN, USA) and a phosphatase inhibitor cocktail (Sigma). The proteins were quantified using a modified Bradford assay (Bio-Rad, Hercules, CA, USA). For analysis of total protein samples, $25 \mu \mathrm{g}$ of proteins were eluted in sample buffer, and loaded in 7.5 or $12 \%$ SDS-PAGE, depending on the proteins molecular weight. The proteins were then electroblotted onto a Hybond ECL membrane (Amersham Biosciences, Buckinghamshire, UK). Membranes were blocked with 5\% non-fat milk or 4\% BSA and $0.5 \%$ Tween-20 in PBS and immunoblotted with antibodies against E-cadherin (1:1000, BD Biosciences), $\beta$-catenin (1:1000, Sigma), p120 (1:1000, BD Biosciences), PIPKI $\gamma$ (1:1000, Cell Signaling Technologies), Hakai (1:500, Zymed Laboratories, Camarillo, CA, USA), $\alpha$-Tubulin (1:10000, Sigma) or Actin (1:1000, Santa Cruz Biotechnology). Donkey anti-rabbit (Amersham Biosciences), sheep anti-mouse (Amersham Biosciences) or donkey anti-goat (Santa Cruz Biotechnology) HRP-conjugated secondary antibodies were used, followed by ECL detection (Amersham Biosciences).

\section{Flow cytometry}

Cells were grown to a confluent monolayer, detached with Versene (Gibco, Invitrogen) and resuspended in ice-cold PBS with $0.05 \mathrm{mg} / \mathrm{ml} \mathrm{CaCl}_{2}$. For all conditions, $5 \times 10^{5}$ cells were centrifuged for $5 \mathrm{~min}$ at 1500 r.p.m. and $4{ }^{\circ} \mathrm{C}$, 
and washed in PBS with $0.05 \mathrm{mg} / \mathrm{ml} \mathrm{CaCl}_{2}$ and $3 \%$ BSA. Cells were incubated for $1 \mathrm{~h}$ with the extracellular primary antibody against E-cadherin, HECD1 (Zymed Laboratories) at 1:50 dilution. Cells were washed twice and incubated with Alexa Fluor 488 goat anti-mouse (1:250, Invitrogen, Eugene, OR, USA) in the dark for $30 \mathrm{~min}$. Finally, the cells were washed and resuspended in $0.5 \mathrm{ml}$ of washing solution. At least $5 \times 10^{4}$ cells were analyzed in a Colter Epics XL-MCL flow cytometer. The data were analyzed with WinMDI software.

\section{Immunofluorescence staining}

Cells were seeded on 6-well plates on top of glass coverslips and grown to at least $80 \%$ confluence. Fixation was performed in ice-cold methanol for $20 \mathrm{~min}$, followed by washing and blocking in 5\% BSA in PBS for $30 \mathrm{~min}$, at room temperature. The mouse monoclonal E-cadherin antibody (BD Biosciences) was used at 1:300 dilution in PBS with BSA 5\% and incubated for $1 \mathrm{~h}$ at room temperature. An Alexa Fluor 488 goat anti-mouse (1:500, Invitrogen) was applied for $1 \mathrm{~h}$ in dark as secondary antibody. The coverslips were mounted on slides using Vectashield with DAPI (Vector Laboratories). Images were acquired on a Carl Zeiss Apotome Axiovert 200M Fluorescence Microscope (Carl Zeiss), using $\times 20$ and $\times 40$ objectives. Images were taken with an Axiocam HRm camera and processed with the Zeiss Axion Vision 4.8 software.

\section{Slow aggregation assay}

The functional significance of the E-cadherin missense mutations was assessed by slow aggregation assay as described in Suriano et al. ${ }^{12}$ Wells of a 96-well-plate were coated with $50 \mu \mathrm{l}$ of an agar solution (100 mg Bacto-Agar in $15 \mathrm{ml}$ of sterile PBS). Cells were detached with trypsin, resuspended in culture medium and $2 \times 10^{4}$ cells were seeded in each well. Experiments always included triplicates. The plate was incubated at $37^{\circ} \mathrm{C}$, in a humidified atmosphere with $5 \% \mathrm{CO}_{2}$, for $48 \mathrm{~h}$. Aggregation was evaluated under an inverted microscope (objective of $\times 4$ ) and photographed with a Nikon (Tokyo, Japan) digital camera.

\section{Matrigel invasion assay}

For invasion assays, 24-well matrigel invasion chambers (BD Biocoat, Erembodegem, Belgium) were hydrated by filling the inner and outer compartments with $\alpha$-MEM medium and incubating them for $1 \mathrm{~h}$ at $37^{\circ} \mathrm{C}$. Cells were detached with trypsin, resuspended in culture medium and $5 \times 10^{4}$ cells were seeded in each chamber. The plate was incubated at $37^{\circ} \mathrm{C}$, in a humidified atmosphere with $5 \% \mathrm{CO}_{2}$ for $24 \mathrm{~h}$. The non-invasive cells and matrigel from the upper side of the filters were removed with a pre-wet 'cotton swab'. The filters were washed in PBS, fixed in ice-cold methanol for $15 \mathrm{~min}$ and mounted in slides with Vectashield with DAPI (Vector Laboratories). The total number of invasive nuclei was counted using a Leica DM2000 microscope (Leica, Cambridge, UK).

\section{Statistical analysis}

Two-tailed paired Student's $t$-test was used to perform statistical analysis. In all analysis $P<0.05$ was required for statistical significance. Statistical analysis was done using StatView software program (PC version).

\section{RESULTS}

Mutations located at p120-catenin binding domain impair the interplay of E-cadherin with p120, allowing the binding of Hakai An important post-translational mechanism of E-cadherin regulation is the endocytic process. ${ }^{23-26}$ This pathway controls the amount of E-cadherin present at the PM and p120 is considered its main regulator. $^{23,24,26,43,44}$ In order to investigate whether E-cadherin mutations could be more unstable at the PM due to the lack of p120-catenin binding and stabilization, we selected and analyzed five cytoplasmic missense mutations - R749W, E757K, E781D, P799R and V832M - and two extracellular missense mutations - T340A and A634V (Figure 1).

A new strategy to drive the expression of the E-cadherin mutants was established. $\mathrm{CHO}$ cells were transfected with the different $\mathrm{CDH} 1$ constructs and were evaluated for the interaction with p120-catenin, using PLA.

The results showed that mutations localized at p120 binding domain (Figure 1) drastically reduce the amount of interactions with p120-catenin, depicted as red dots (PLA signals) in Figure 2a. The quantification of the number of PLA signals (Figure $2 b$ ) demonstrated that R749W leads to a reduction of 78\% in the number of PLA signals (from 1.00 to $0.22, P=0.00071$ ), E757K exhibited a reduction of $64 \%$ (from 1.00 to $0.36, P=0.0093$ ) and the mutant E781D 69\% (from 1.00 to $0.31, P=0.0016$ ). The remaining mutants also have a slight decrease in the number of interactions, suggesting that mutant proteins are less stable at PM. Western blot results prove that these differences are not justified by protein expression differences (Figure 2c).

It is well known that E-cadherin is internalized by endocytosis when p120 dissociates from the juxtamembrane domain of E-cadherin and ubiquitin ligase Hakai is a key factor in the demise of p120deprived E-cadherin. ${ }^{29,30,33}$ Therefore, we investigated whether loss of p120 association with mutant E-cadherin lead to increased interaction with Hakai. Although the expression levels of Hakai remain unchanged (Figure 3c), we verified that E-cadherin mutations that block p120 association, those located at p120 binding domain, resulted in a significant increase of the interplay with Hakai: 1.84fold for R749W $(P=0.027), 2.29$-fold for E757K and 1.73-fold for E781D $(P=0.036)$ (Figures $3 \mathrm{a}$ and $\mathrm{b})$. As expected, the other mutants also allowed a small increase of the E-cadherin/Hakai interaction 1.41 for T340A, 1.45 for A634V, 1.35 for P799R and 1.24 for V832M as these mutated proteins lead to a slight dissociation of p120 (Figure 2b). Taken together, these results indicate that all the analyzed E-cadherin missense mutations are less stable at the PM, and probably result in higher endocytosis.

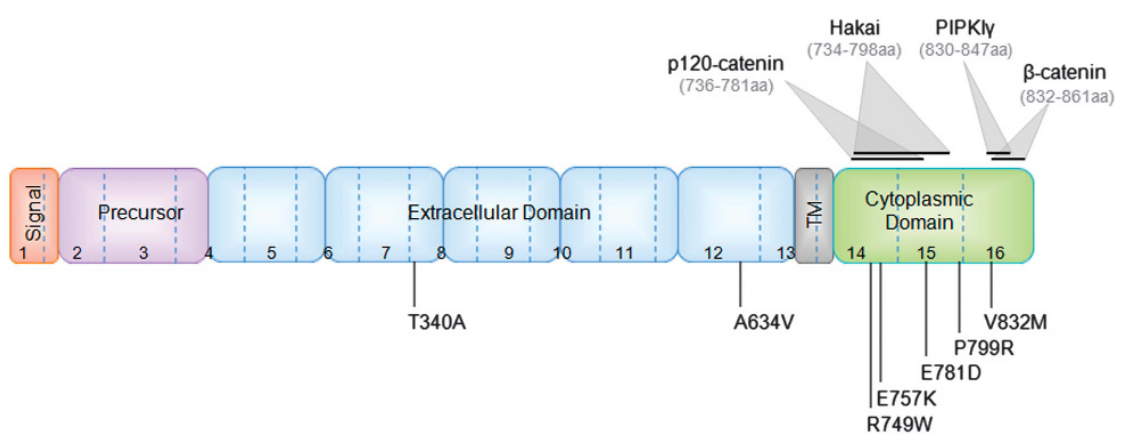

Figure 1 Illustration of the E-cadherin missense mutations' location and the binding region of E-cadherin partners. Location of the germline E-cadherin mutations, identified in HDGC families, used in this study. The location of the protein signal peptide, precursor sequence, extracellular domain, transmembrane domain (TM) and cytoplasmic domain, as well as the binding domain of p120, Hakai, PIPKI $\gamma$ and $\beta$-catenin are represented. 

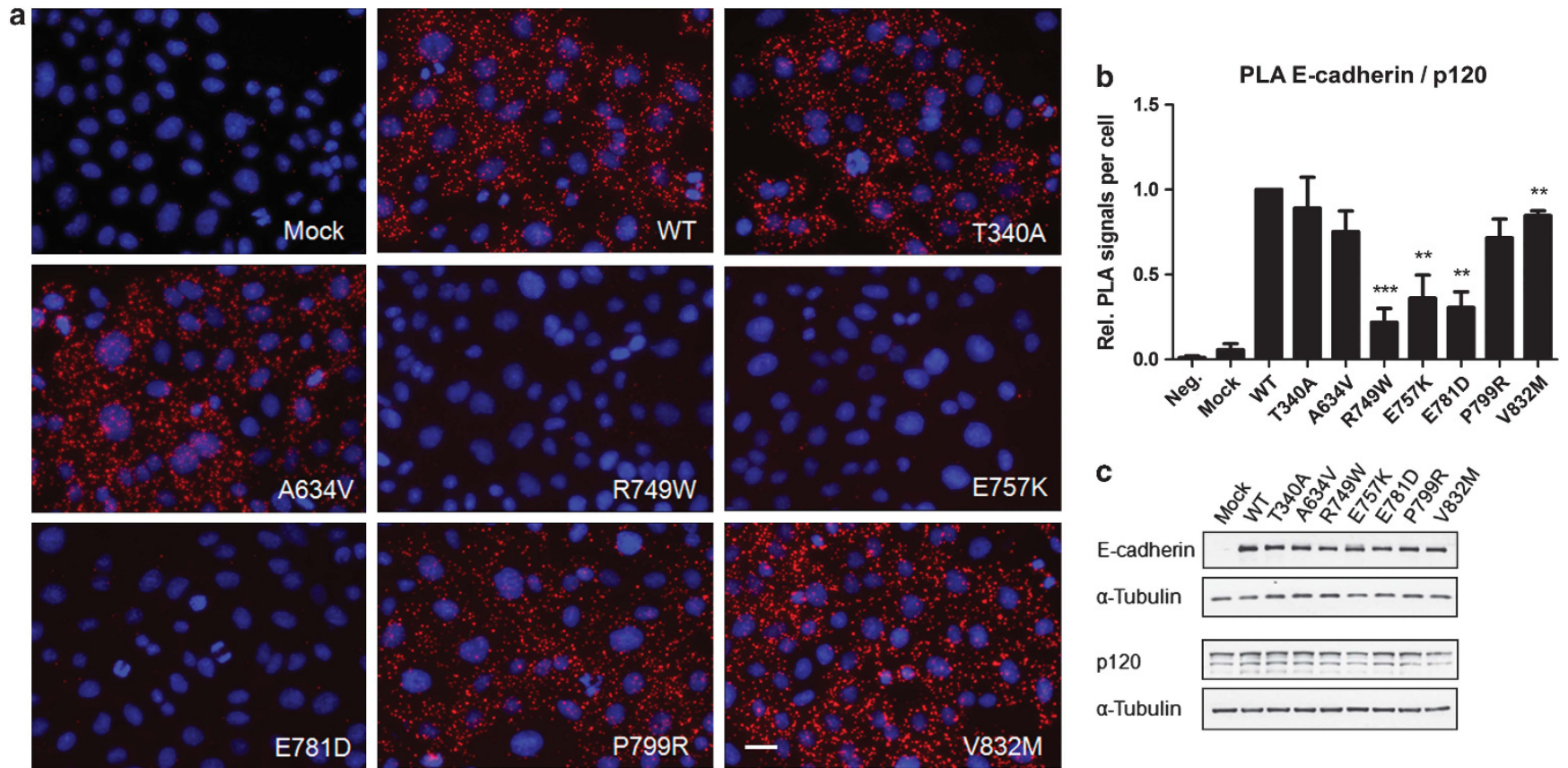

Figure 2 Changes in the p120 binding domain are dramatic for E-cadherin and p120-catenin association. (a) The interaction between WT or mutant E-cadherin and p120-catenin was assessed by in situ PLA. CHO cells stably transfected with the empty vector (Mock) or with WT, T340A, A634V, R749W, E757K, E781D, P799R or V832M hE-cadherin were fixed and incubated with antibodies against E-cadherin and p120-catenin. In the negative control (Neg.), CHO WT cells were incubated only with the antibody against E-cadherin. Close proximity of oligonucleotide-ligated secondary antibodies allows rolling-circle amplification and detection of the amplification product by a fluorescence labeled probe (red dots). Nuclei were counterstained with DAPI (blue). The pictures were taken under a $\times 40$ objective. Scale bar represents $20 \mu \mathrm{m}$. (b) The number of PLA signals per cell was quantified in each condition. The graph shows the average of relative number of PLA signals per cell $\pm \mathrm{SE}, n=3\left({ }^{*} P \leq 0.01\right.$ and $\left.* * * P \leq 0.001\right)$. (c) E-cadherin, $p 120$-catenin and $\alpha$-Tubulin expression levels were detected in whole-cell lysates by western blot. $\alpha$-Tubulin was used as a loading control. The images shown are representative of three independent experiments.
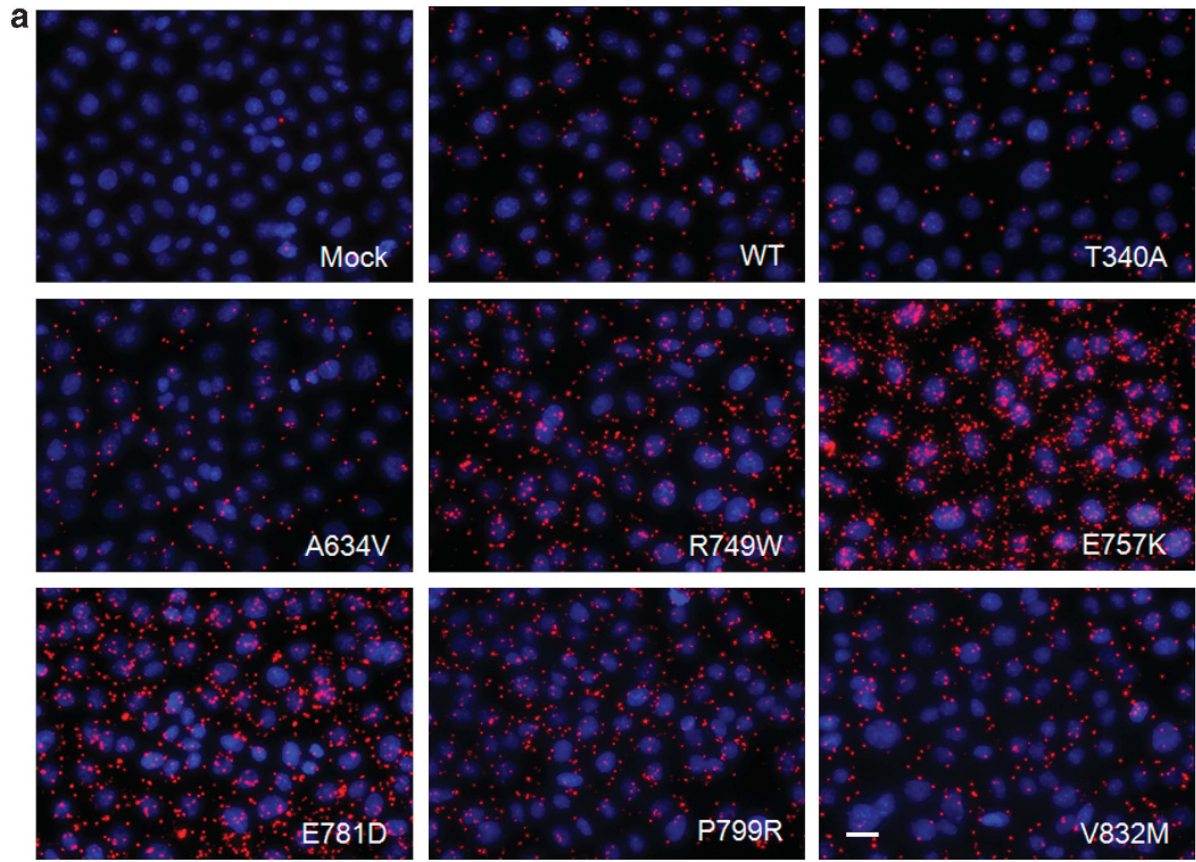

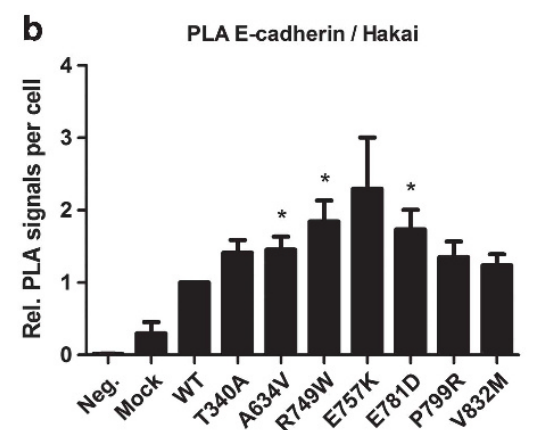

C

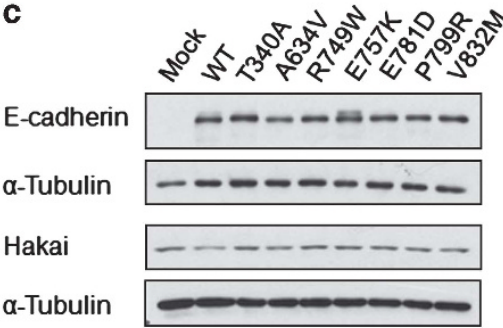

Figure 3 E-cadherin deprived of p120-catenin is more susceptible to Hakai association. (a) The interplay of WT and mutant E-cadherin with Hakai was assessed by in situ PLA. Close proximity of these two proteins gives rise to PLA signals (red dots). Nuclei were counterstained with DAPI (blue). Scale bar represents $20 \mu \mathrm{m}$. (b) The graph shows the average of the relative number of PLA signals per cell $\pm \mathrm{SE}, n=4$ ( ${ }^{*} \leq 0.05$ ). (c) Western blot was used to assess E-cadherin, Hakai and $\alpha$-Tubulin expression levels. 

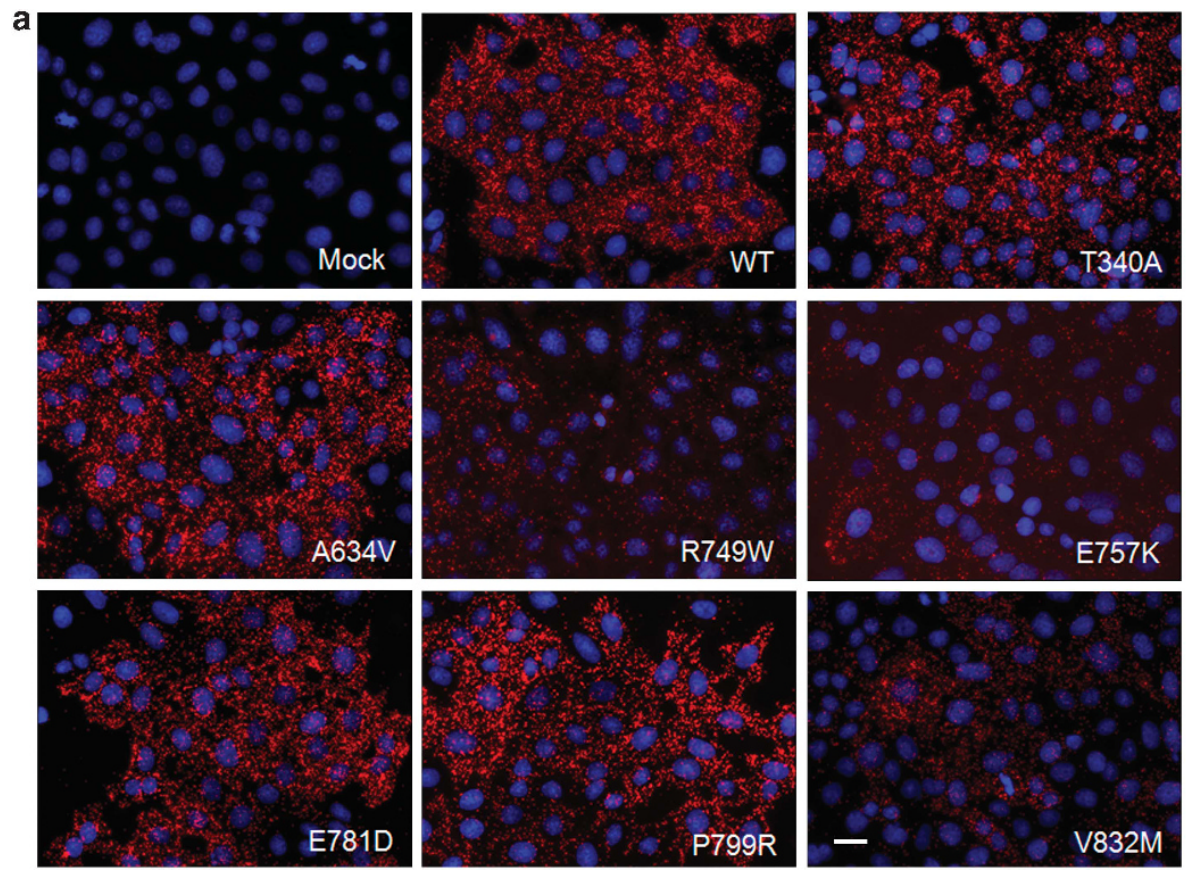

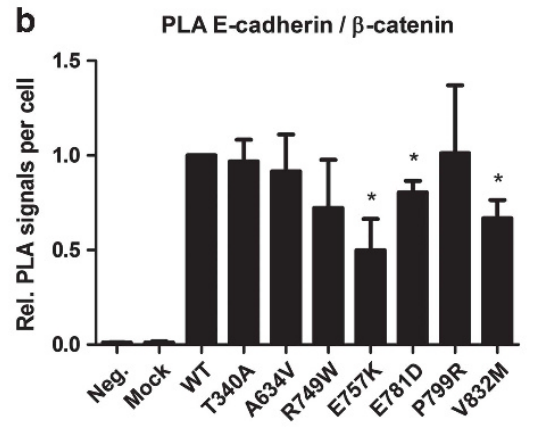

c

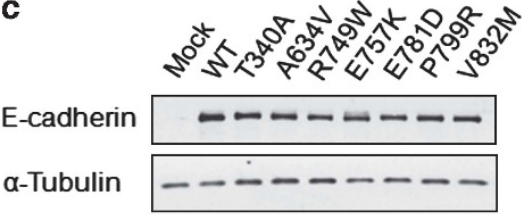

$\beta$-Catenin

$\alpha$-Tubulin

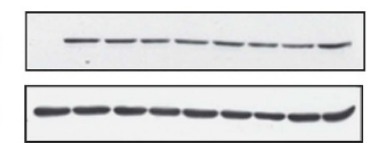

Figure 4 E-cadherin cytoplasmic mutations affect the interaction with $\beta$-catenin. (a) The interaction between WT or mutant E-cadherin and $\beta$-catenin was analyzed by PLA. Red dots indicate PLA signals and nuclei were counterstained with DAPI (blue). Scale bar represents $20 \mu \mathrm{m}$. (b) The number of PLA signals per cell was quantified in each condition. The graph shows the relative number of PLA signals per cell $\pm \mathrm{SE}, n=3\left({ }^{*} P \leq 0.05\right)$. (c) E-cadherin, $\beta$-catenin and $\alpha$-Tubulin were detected in whole-cell lysates by western blot.

E-cadherin cytoplasmic mutations interfere with the binding of key exocytosis-related partners

Knowing that $\beta$-catenin and PIPKI $\gamma$ have crucial roles on E-cadherin exocytosis and function and both bind directly to E-cadherin, ${ }^{23,24,28,45-47}$ we used PLA to evaluate whether the mutations interfere with this interplay, leading to E-cadherin trafficking deregulation and, consequently, loss of function.

We could verify that, when compared with WT, the mutants R749W, E757K, E781D and V832M present a reduced interaction E-cadherin/ $\beta$-catenin (Figures $4 \mathrm{a}$ and b). The mutants R749W, E757K, E781D and V832M have, respectively, 0.72, 0.50, 0.80 and 0.67 fold change of the WT protein interactions, and this decrease is statistically significant for the mutants E757K $(P=0.039)$, E781D $(P=0.032)$ and V832M $(P=0.026)$.

Analyzing the data of PIPKI $\gamma$ interaction (Figure 5), we verified that all mutants, with the exception of the mutant T340A, have a decreased E-cadherin/PIPKI $\gamma$ interplay - 1.00 in T340A, 0.87 in A634V, 0.73 in R749W, 0.57 in E757K, 0.44 in E781D, 0.51 in P799R and 0.71 in V832M (Figures $5 \mathrm{a}$ and $\mathrm{b}$ ). This decrease is especially prominent in E757K $(P=0.025)$, E781D and P799R $(P=0.028)$ -expressing cells.

The results show that $\mathrm{CDH} 1$ missense mutants lead to a deficient binding of exocytosis-related partners to E-cadherin, and this can compromise the number of E-cadherin molecules that are trafficked to the PM.

CDH1 missense mutations affect E-cadherin subcellular localization as a result of trafficking deregulation

To clarify whether different interaction abilities (of WT and mutant proteins) result in abnormal E-cadherin localization and expression patterns, we decide to perform immunofluorescence and flow cytometry analysis.
As shown in Figure 6a, WT E-cadherin is correctly located at PM, as well as the mutant T340A. The mutants A634V, R749W, E757K, E781D, P799R and V832M present reduced E-cadherin expression at the membrane and aberrant cytoplasmic accumulation.

Flow cytometry results (Figures $6 \mathrm{~b}-\mathrm{d}$ ) showed that within the set of mutations tested, the cells expressing the mutants E781D, P799R and V832M present less number of cells positive for membrane E-cadherin and this difference is statistically significant for the mutants E781D and P799R ( $P=0.053$ and $P=0.0006$, respectively). Considering fluorescence intensity (Figure 6d), the mutants A634V, R749W, E757K and P799R generated reduced staining intensity, meaning that less E-cadherin molecules are present at the PM: $0.40(P=0.044)$, $0.53(P=0.0021), 0.62(P=0.017)$ and $0.64(P=0.013)$, respectively.

Cytoplasmic E-cadherin missense mutations are functionally relevant as they have deficient adhesion and are invasive In order to test the biological relevance of our findings, we functionally tested all E-cadherin mutants, using the in vitro classical assays (slow aggregation and matrigel invasion assays), to evaluate the pathogenic significance of $\mathrm{CDH} 1$ missense mutations.

We observed that all the mutants were functionally relevant, which is in accordance with other reports. ${ }^{5,12,15-19,21}$ The slow aggregation assay showed that all the mutants have an impact on the cell-cell adhesion ability but the type of aggregation showed distinct phenotypes (Figure 7a). Comparing with the compact aggregates formed by the WT E-cadherin-expressing cells, some mutants display smaller cellular aggregates, such as mutants T340A, A634V, R749W, P799R and V832M, and others exhibited a close-to-isolated phenotype, as observed for mutants E757K and E781D, very similar to the phenotype of Mock cells.

As expected, the invasion assay showed that all the mutants were more invasive than the WT-expressing cells (Figure 7b) but, 

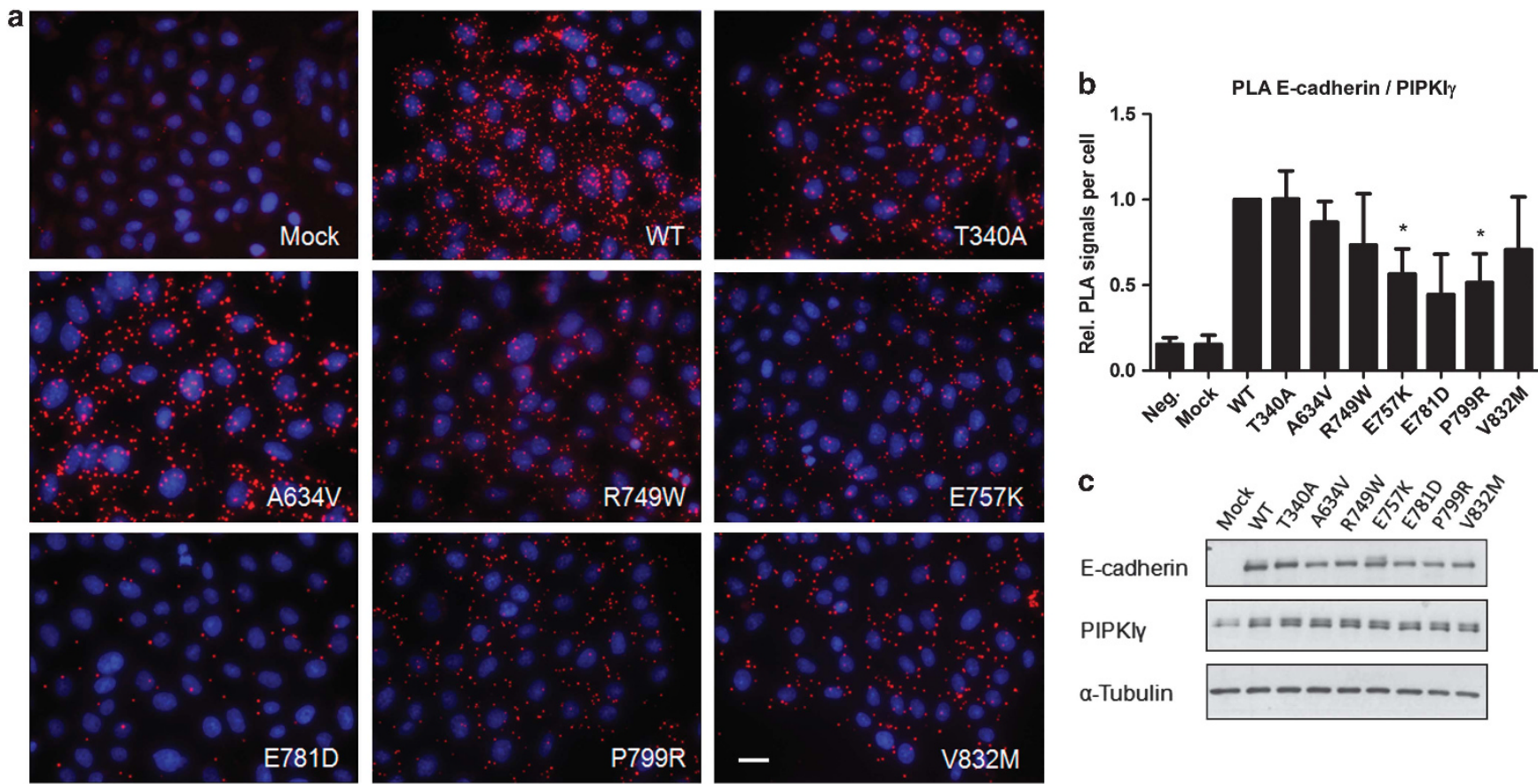

C

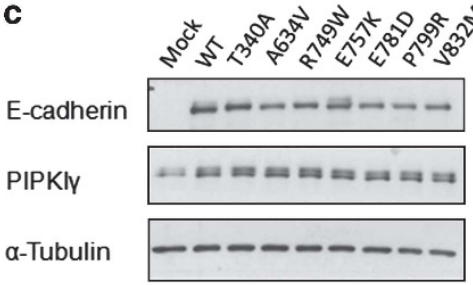

Figure 5 E-cadherin mutants have a decreased E-cadherin/PIPKI interplay. (a) Association of WT or mutant E-cadherin with PIPKI (red dots). Nuclei were counterstained with DAPI (blue). Scale bar represents $20 \mu \mathrm{m}$. (b) The number of PLA signals per cell was quantified in each condition (*P $\leq 0.05$ ). (c) Protein expression levels of E-cadherin, PIPKI $\gamma$ and $\alpha$-Tubulin were analyzed by western blot.

interestingly, the invasion ability of the cytoplasmic mutants is significantly less evident $(P=0.017)$. Therefore, the use of PLA to study the ability of cytoplasmic E-cadherin mutants to interact with its binding partners is of critical importance to complement the functional assays currently used to evaluate the pathogenic significance of E-cadherin mutations associated to HDGC.

\section{DISCUSSION}

E-cadherin cytoplasmic domain has a crucial role for E-cadherin function because it supports the assembly of a complex of cytosolic proteins, including $\alpha_{-}, \beta-,, \mathrm{p} 120-$ and $\gamma$-catenins, which provide the anchorage to the actin cytoskeleton to form a stable cell-cell contact. ${ }^{45,46}$ This domain also has an essential role for protein trafficking and regulation at the $\mathrm{PM} .^{23-26}$ The association of $\beta$-catenin and PIPKI $\gamma$ to E-cadherin cytoplasmic tail is necessary for newly synthesized E-cadherin delivery to basal-lateral membrane. ${ }^{27,28}$ At the PM, E-cadherin molecules constitutively undergo endocytosis and can either be recycled back to the PM or be degraded. ${ }^{23}$ The p120-catenin is the best known inhibitor of cadherin endocytosis, as its binding to the E-cadherin juxtamembrane domain is required for maintenance and stability of E-cadherin molecules at the PM and, simultaneously, it physically blocks the interaction with proteins from the endocytic machinery, such as clathrin adaptor proteins and Hakai. ${ }^{29-33}$ Importantly, Hakai binds directly to E-cadherin and, being an E3 ubiquitin ligase, it ubiquitinates and induces E-cadherin endocytosis. ${ }^{33}$

Taking into account the present knowledge about E-cadherin regulation, we tested whether and how the E-cadherin cytoplasmic mutations interfere with key trafficking-related partners, leading to abnormal E-cadherin expression, localization and function, supporting their pathogenic relevance.

For this purpose, a number of E-cadherin mutations associated to HDGC was explored (Figure 1). As first step, we have studied the above mentioned interactions using PLA, a recent proteomic tool to detect protein-protein interactions. ${ }^{39,40}$

We were able to demonstrate that the association of p120-catenin with E-cadherin directly depends on its binding domain. We are showing for the first time, that HDGC-associated mutations affecting the p120 binding domain (R749W, E757K and E781D) result in a dramatic impact in the number of molecules that are in complex with this partner and, as consequence, these mutants are more available to be targeted by Hakai for ubiquitination and to be degraded (Figures 2 and 3).

The analysis of PLA for E-cadherin/ $\beta$-catenin (Figure 4 ) shows that E-cadherin mutations can affect this interaction, even in the case of mutations located outside the $\beta$-catenin binding domain, as we have previously demonstrated for mutants R749W and E757K. ${ }^{21,47}$ Surprisingly, the mutant V832M, which was described to interact normally with $\beta$-catenin but not with $\alpha$-catenin, ${ }^{36,48}$ shows decreased association with $\beta$-catenin. This can be due to the increased sensibility and accuracy of PLA to detect protein-protein interactions when compared with co-immunoprecipitation technique. Co-immunoprecipitation experiments are much more difficult to perform and present several disadvantages such as nonspecific ligation to IP-beads, decreased affinity for interaction in detergent lysates and the need to use huge sample amounts.

The interplay of E-cadherin mutants with PIPKI $\gamma$ was also analyzed and clearly demonstrate that all intracellular mutants display decreased ability to interact with PIPKI $\gamma$ (Figure 5). It seems that the association of these two proteins is very sensitive to structural alterations of E-cadherin cytoplasmic tail. Interestingly, the mutants E757K, E781D and P799R seem to have a more deleterious effect than the mutant V832M, which is located within the PIPKI $\gamma$ binding region and was described to interfere with this interaction. ${ }^{28}$

To understand whether a decreased interaction of E-cadherin with p120, $\beta$-catenin and PIPKI $\gamma$, and an increased interaction with Hakai 
a
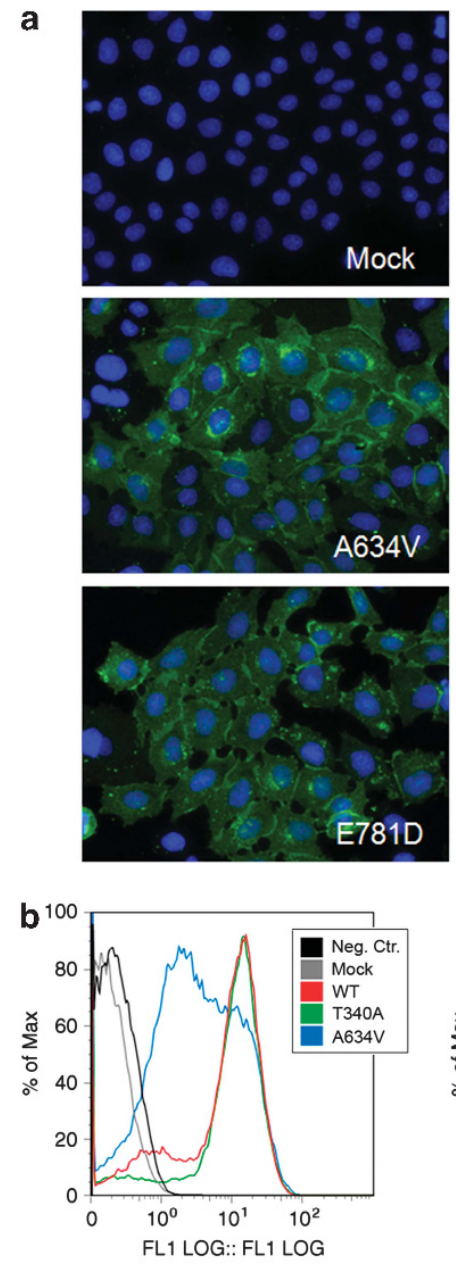

C

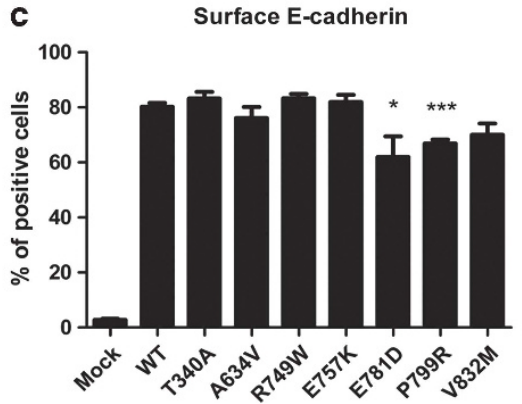

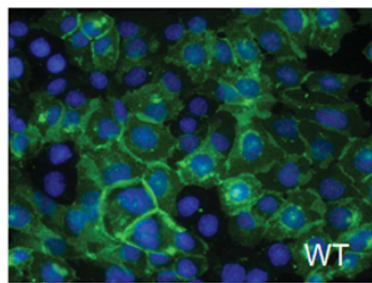
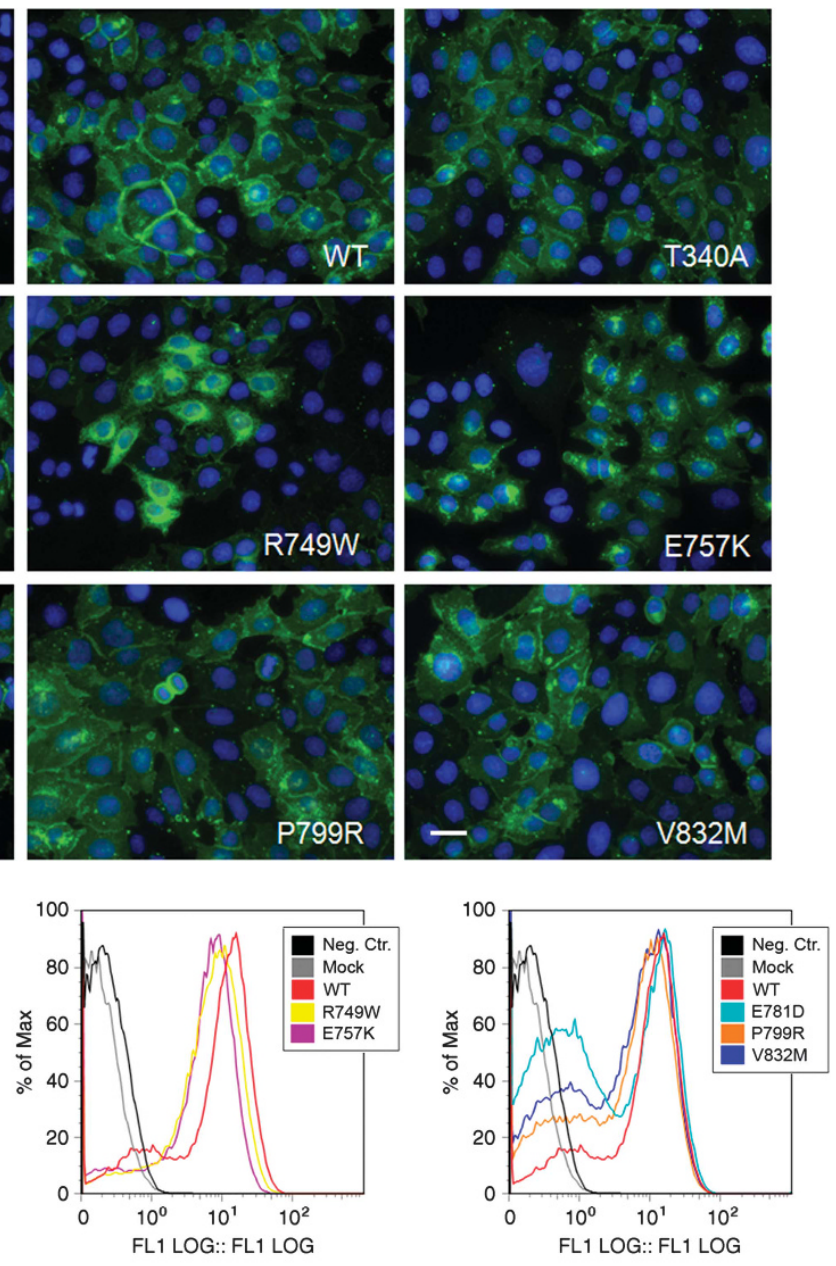

d

Fluorescence Intensity

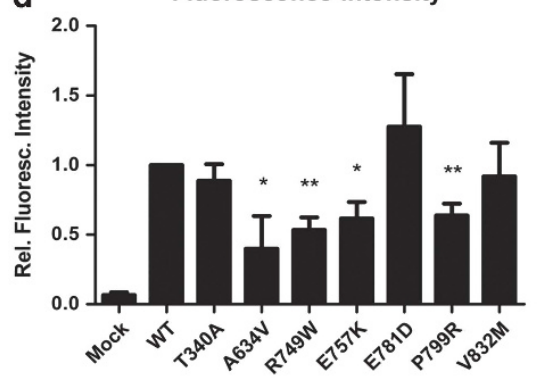

Figure $6 \mathrm{E}$-cadherin missense mutations affect the subcellular localization, as a result of trafficking deregulation. (a) $\mathrm{CHO}$ cells stably transfected with the empty vector (Mock) or with WT, T340A, A634V, R749W, E757K, E781D, P799R or V832M hE-cadherin were fixed and immunostained with anti-human E-cadherin antibody (green). Nuclei were counterstained with DAPI (blue). Scale bar represents $20 \mu \mathrm{m}$. (b) Flow cytometry technique was used to assess cell surface E-cadherin. Each histogram represents surface E-cadherin expression in Mock cells, in WT-expressing cells and in extracellular/juxtamembrane/ cytoplasmic mutations. The black line in the histograms represents the WT cells that were not incubated with primary antibody, this sample was used as negative control. (c) For each sample, the mean of cells expressing surface E-cadherin was calculated. The graph shows the average \pm SE of four independent experiments. (d) The mean fluorescence intensity was also quantified and normalized for cells expressing WT E-cadherin (* $P \leq 0.05$, ${ }^{* *} P \leq 0.01$ and $\left.{ }^{* * *} P \leq 0.001\right)$.

could reflect deficient E-cadherin surface expression and abnormal localization, we analyzed both parameters. We have found that other mutations, in addition to R749W and $\mathrm{E} 757 \mathrm{~K},{ }^{21}$ can affect E-cadherin localization and surface expression. The mutants A634V, E781D, P799R and V832M also present less staining at the PM and increased cytoplasmic accumulation of E-cadherin (Figure 6). Indeed, it was already described by other group that the V832M mutant, besides being present at the PM as described by our group, ${ }^{15}$ is also largely accumulated in the cytosolic compartment. ${ }^{28}$

Our results suggest that E-cadherin structural alterations decrease the affinity of exocytic partners to bind to E-cadherin molecules. We have previously shown that a great fraction of E-cadherin mutant protein is recognized as unfolded and is degraded by the endoplasmic reticulum quality control. ${ }^{21}$ However, some E-cadherin molecules are 

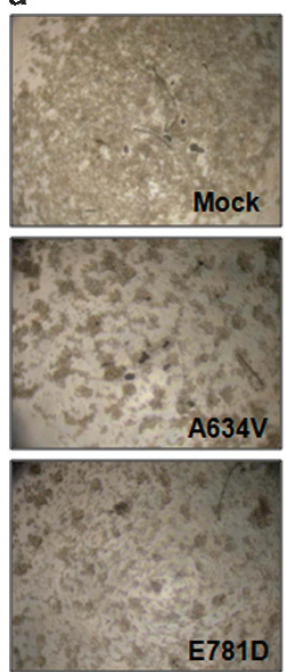
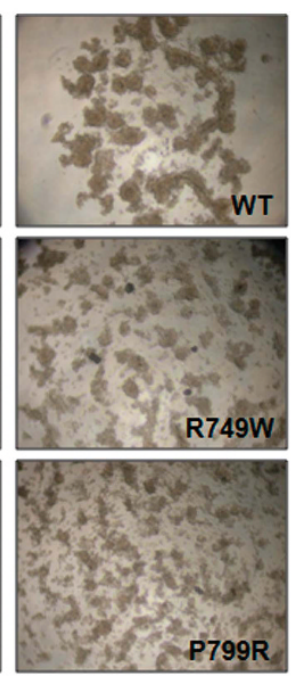
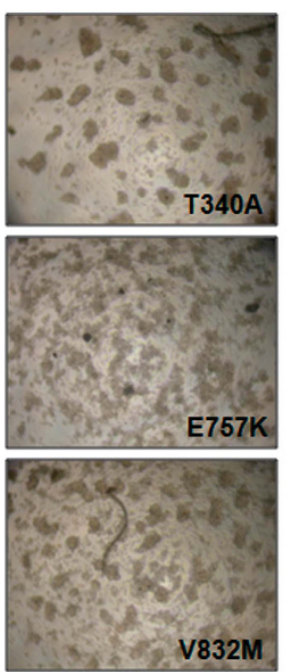

b

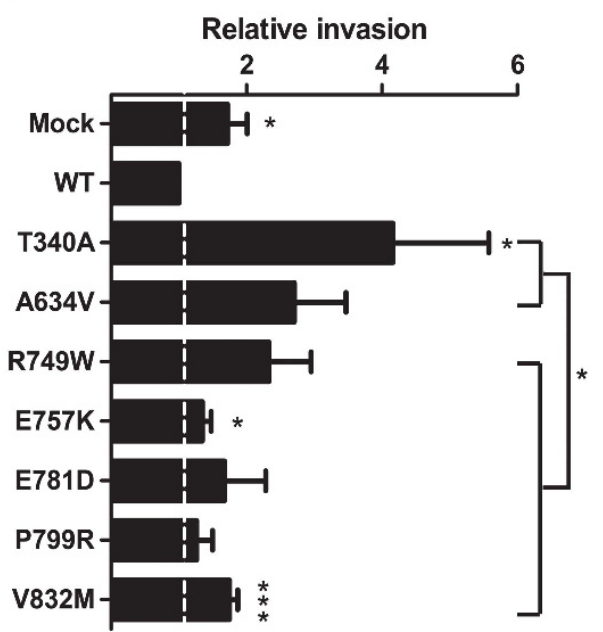

Figure 7 Cytoplasmic E-cadherin missense mutations are less invasive than the extracellular ones. (a) CHO cells stably transfected with the empty vector (Mock), WT E-cadherin or different mutants were analyzed for cell-cell aggregation ability by slow aggregation assays. The images shown are representative of three independent experiments. (b) For each mutant cell line, matrigel invasion assays were performed. The graph shows the relative number of invasive cells $\pm \mathrm{SE}$ of four independent experiments $\left({ }^{*} P \leq 0.05\right.$ and $\left.* * * P \leq 0.001\right)$.

able to interact with these partners and are transported to the PM, escaping to ERAD. At the cell membrane, the cell has other mechanisms to abolish abnormal E-cadherin forms. Abnormal E-cadherin does not establish a strong enough contact with catenins, and proteins from the endocytic machinery have the opportunity to bind, initiating the endocytosis process that can culminate in E-cadherin degradation.

Importantly, our present findings corroborate a recent work of our laboratory where we demonstrated, by in silico and in vitro approaches, that specific E-cadherin mutations, no matter being extracellular or intracellular, lead to protein destabilization and, as consequence, premature degradation. ${ }^{49}$ For all these reasons, quantification of E-cadherin present at the PM, as well as its subcellular localization, should be analyzed for all missense mutants found in HDGC.

Finally, we confirmed that all seven HDGC missense mutations chosen herein impaired cell-cell adhesion and induced invasion (Figure 7). Regarding invasion, the pathogenic behavior of extracellular mutations is more exuberant than E-cadherin cytoplasmic mutations. In fact, we have previously shown a genotype-phenotype correlation between the localization of $\mathrm{CDH} 1$ missense mutations and their in vitro functional behavior. ${ }^{50}$ Cells expressing E-cadherin mutations located at the extracellular domain are more motile, conferring a more aggressive in vitro phenotype, than those affecting the E-cadherin intracellular portion. ${ }^{36,50}$ For that reason, E-cadherin mutations localized at the intracellular domain pose additional problems in clinical terms and make it urgent to improve the accuracy of the in vitro methods. Being aware of this, the structural E-cadherin model was recently updated and now covers the prodomain, the extracellular domain and the catenin binding domain. ${ }^{49}$ Nevertheless, predictive tests are limited due to the small number of $\mathrm{CDH} 1$ germline missense variants available, and to the lack of structural information for some portions of the protein, such as the juxtamembrane domain.

Hence, it would be very helpful to use new complementary tools, such as PLA, to improve the classification of the cytoplasmic $\mathrm{CDH} 1$ missense mutations as pathogenic or non-pathogenic. Accordingly, we could observe a correlation between PLA studies and E-cadherin adhesiveness. We verified that the mutants impairing concomitantly the binding of $\mathrm{p} 120, \beta$-catenin and PIPKI $\gamma$, had the strongest impact on adhesive properties, as observed for the mutants E757K and E781D, which displayed a close-to-isolated phenotype. In contrast, the mutant T340A, which only slightly affected the interplay with p120catenin, presented a mild effect on cell aggregation, emphasizing the importance of these partners for E-cadherin-mediated cell-cell adhesion. The remaining mutants presented an in-between phenotype, as they have a reduced interplay with two of the binding partners.

Despite not being a direct observation of E-cadherin trafficking, the study of the interplay between E-cadherin and its binding partners is indicative about the assembly of E-cadherin-catenins complex and, consequently, of E-cadherin functionality. Thus, if the assembly of the cytoplasmic complex is compromised, E-cadherin is not able to form a stable cell-cell contact and to mediate cell adhesion, resulting probably in increased E-cadherin turnover and degradation.

Furthermore, our results suggest that each mutation behaves in a singular way as it interacts differently with its binding partners, reflects its particular phenotype and possibly has different roles in signal transduction. For these reasons, we believe that each E-cadherin missense mutation is likely to induce cell-specific biological behavior with distinct clinical impact within HDGC.

In this paper, we show that PLA is a powerful method to evaluate the ability of E-cadherin mutants to interact with its direct binding partners. Besides being a quantitative method, it is easy and fast to perform, and these conditions are essential to be applied in routine. In addition, the set of interactions chosen for this analysis cover most of the cytoplasmic tail of E-cadherin, making possible to infer the functional relevance of mutations affecting this domain. Using this technique, we can offer a more accurate and robust evaluation of mutation pathogenic significance for diagnostic, especially in the case of intracellular missense mutations.

\section{CONFLICT OF INTEREST}

The authors declare no conflict of interest. 


\section{ACKNOWLEDGEMENTS}

This work was supported by grants from the Fundação para a Ciência e Tecnologia, Portugal (PTDC/SAU-OBD/104017/2008, PTDC/SAU-ONC/ $110294 / 2009$ and SFRH/BD/43763/2008). IPATIMUP is an Associated Laboratory of the Portuguese Ministry of Science and Technology and Higher Education and is partially supported by FCT.

1 Lauren P: The two histological main types of gastric carcinoma: diffuse and so-called intestinal-type carcinoma. An attempt at a histo-clinical classification. Acta Pathol Microbiol Scand 1965; 64: 31-49.

2 Guilford PJ, Hopkins JB, Grady WM et al: E-cadherin germline mutations define an inherited cancer syndrome dominated by diffuse gastric cancer. Hum Mutat 1999; 14: 249-255.

3 Oliveira C, Seruca R, Carneiro F: Hereditary gastric cancer. Best Pract Res Clin Gastroenterol 2009; 23: 147-157.

4 Keller G, Vogelsang H, Becker I et al: Diffuse type gastric and lobular breast carcinoma in a familial gastric cancer patient with an E-cadherin germline mutation. Am J Pathol 1999; 155: 337-342.

5 Brooks-Wilson AR, Kaurah P, Suriano G et al: Germline E-cadherin mutations in hereditary diffuse gastric cancer: assessment of 42 new families and review of genetic screening criteria. J Med Genet 2004; 41: 508-517.

6 Schrader KA, Masciari S, Boyd N et al: Hereditary diffuse gastric cancer: association with lobular breast cancer. Fam Cancer 2008; 7: 73-82.

7 Guilford P, Hopkins J, Harraway J et al: E-cadherin germline mutations in familial gastric cancer. Nature 1998; 392: 402-405.

8 Corso G, Marrelli D, Pascale V, Vindigni C, Roviello F: Frequency of $\mathrm{CDH} 1$ germline mutations in gastric carcinoma coming from high- and low-risk areas: metanalysis and systematic review of the literature. BMC cancer 2012; 12: 8 .

9 Karam R, Carvalho J, Bruno I et al: The NMD mRNA surveillance pathway downregulates aberrant $\mathrm{E}$-cadherin transcripts in gastric cancer cells and in $\mathrm{CDH} 1$ mutation carriers. Oncogene 2008; 27: 4255-4260.

10 Fitzgerald RC, Caldas C: Clinical implications of E-cadherin associated hereditary diffuse gastric cancer. Gut 2004; 53: 775-778.

11 Carneiro F, Oliveira C, Suriano G, Seruca R: Molecular pathology of familial gastric cancer, with an emphasis on hereditary diffuse gastric cancer. J Clin Pathol 2008; 61 . 25-30.

12 Suriano G, Oliveira C, Ferreira $\mathrm{P}$ et al: Identification of $\mathrm{CDH} 1$ germline missense mutations associated with functional inactivation of the E-cadherin protein in young gastric cancer probands. Hum Mol Genet 2003; 12: 575-582.

13 Suriano G, Seixas S, Rocha J, Seruca R: A model to infer the pathogenic significance of $\mathrm{CDH} 1$ germline missense variants. J Mol Med 2006; 84: 1023-1031.

14 van Roy F, Berx G: The cell-cell adhesion molecule E-cadherin. Cell Mol Life Sci 2008. 65: 3756-3788

15 Suriano G, Mulholland D, de Wever 0 et al: The intracellular E-cadherin germline mutation V832 M lacks the ability to mediate cell-cell adhesion and to suppress invasion. Oncogene 2003; 22: 5716-5719.

16 Keller G, Vogelsang H, Becker I et al: Germline mutations of the E-cadherin $(\mathrm{CDH} 1)$ and TP53 genes, rather than of RUNX3 and HPP1, contribute to genetic predisposition in German gastric cancer patients. J Med Genet 2004; 41: e89.

17 Suriano G, Yew S, Ferreira $P$ et al: Characterization of a recurrent germ line mutation of the E-cadherin gene: implications for genetic testing and clinical management. Clin Cancer Res 2005; 11: 5401-5409.

18 Kaurah $\mathrm{P}$, MacMillan A, Boyd $\mathrm{N}$ et al: Founder and recurrent $\mathrm{CDH} 1$ mutations in families with hereditary diffuse gastric cancer. JAMA 2007; 297: 2360-2372.

19 More $\mathrm{H}$, Humar $\mathrm{B}$, Weber $\mathrm{W}$ et al: Identification of seven novel germline mutations in the human E-cadherin (CDH1) gene. Hum Mutat 2007; 28: 203.

20 Corso G, Roviello F, Paredes J et al: Characterization of the P373L E-cadherin germline missense mutation and implication for clinical management. Eur J Surg Oncol 2007; 33: 1061-1067.

21 Simoes-Correia J, Figueiredo J, Oliveira C et al: Endoplasmic reticulum quality control: a new mechanism of $\mathrm{E}$-cadherin regulation and its implication in cancer. Hum $\mathrm{Mol}$ Genet 2008; 17: 3566-3576.

22 Kluijt I, Siemerink EJ, Ausems MG et al: $\mathrm{CDH} 1$-related hereditary diffuse gastric cancer syndrome: Clinical variations and implications for counseling. Int J Cance 2012; 131: 367-376.
23 Bryant DM, Stow JL: The ins and outs of E-cadherin trafficking. Trends Cell Biol 2004; 14: 427-434.

24 Delva E, Kowalczyk AP: Regulation of cadherin trafficking. Traffic 2009; 10 : 259-267.

25 Yap AS, Crampton MS, Hardin J: Making and breaking contacts: the cellular biology of cadherin regulation. Curr Opin Cell Biol 2007; 19: 508-514.

26 D'Souza-Schorey C: Disassembling adherens junctions: breaking up is hard to do. Trends Cell Biol 2005; 15: 19-26.

27 Chen YT, Stewart DB, Nelson WJ: Coupling assembly of the E-cadherin/beta-catenin complex to efficient endoplasmic reticulum exit and basal-lateral membrane targeting of E-cadherin in polarized MDCK cells. J Cell Biol 1999; 144: 687-699.

28 Ling K, Bairstow SF, Carbonara C, Turbin DA, Huntsman DG, Anderson RA: Type I gamma phosphatidylinositol phosphate kinase modulates adherens junction and E-cadherin trafficking via a direct interaction with mu $1 \mathrm{~B}$ adaptin. J Cell Biol, 2007; 176: 343-353.

29 Davis MA, Ireton RC, Reynolds AB: A core function for p120-catenin in cadherin turnover. J Cell Biol 2003; 163: 525-534.

30 Xiao K, Allison DF, Buckley KM et al: Cellular levels of p120 catenin function as a set point for cadherin expression levels in microvascular endothelial cells. J Cell Biol 2003; 163: 535-545.

31 Ireton RC, Davis MA, van Hengel J et al: A novel role for p120 catenin in E-cadherin function. J Cell Biol 2002; 159: 465-476.

32 Xiao K, Garner J, Buckley KM et al: p120-Catenin regulates clathrin-dependent endocytosis of VE-cadherin. Mol Biol Cell 2005; 16: 5141-5151.

33 Fujita $Y$, Krause G, Scheffner $M$ et al: Hakai, a c-Cbl-like protein, ubiquitinates and induces endocytosis of the E-cadherin complex. Nat Cell Biol 2002; 4: 222-231.

34 Oliveira C, Bordin MC, Grehan N et al: Screening E-cadherin in gastric cancer families reveals germline mutations only in hereditary diffuse gastric cancer kindred. Hum Mutat 2002; 19: 510-517.

35 Zhang Y, Liu X, Fan Y et al: Germline mutations and polymorphic variants in MMR, $\mathrm{E}$-cadherin and MYH genes associated with familial gastric cancer in Jiangsu of China. Int J Cancer 2006; 119: 2592-2596.

36 Suriano G, Oliveira MJ, Huntsman D et al: E-cadherin germline missense mutations and cell phenotype: evidence for the independence of cell invasion on the motile capabilities of the cells. Hum Mol Genet 2003; 12: 3007-3016.

37 Oliveira C, Ferreira P, Nabais S et al: E-Cadherin (CDH1) and p53 rather than SMAD4 and Caspase-10 germline mutations contribute to genetic predisposition in Portuguese gastric cancer patients. Eur J Cancer 2004; 40: 1897-1903.

38 Yabuta T, Shinmura K, Tani M et al: E-cadherin gene variants in gastric cancer families whose probands are diagnosed with diffuse gastric cancer. Int J Cancer 2002; 101: 434-441.

39 Soderberg 0 , Gullberg M, Jarvius $\mathrm{M}$ et al: Direct observation of individual endogenous protein complexes in situ by proximity ligation. Nat Methods 2006; 3: 995-1000.

40 Weibrecht I, Leuchowius KJ, Clausson CM et al: Proximity ligation assays: a recent addition to the proteomics toolbox. Expert Rev Proteomics 2010; 7: 401-409.

41 Wang J, Wilkinson MF: Site-directed mutagenesis of large (13-kb) plasmids in a single-PCR procedure. Biotechniques 2000; 29: 976-978.

42 Allalou A, Wahlby C: BlobFinder, a tool for fluorescence microscopy image cytometry. Comp Methods Prog Biomed 2009; 94: 58-65.

43 Mosesson Y, Mills GB, Yarden Y: Derailed endocytosis: an emerging feature of cancer. Nat Rev Cancer 2008; 8: 835-850.

44 Xiao K, Oas RG, Chiasson CM, Kowalczyk AP: Role of p120-catenin in cadherin trafficking. Biochim Biophys Acta 2007; 1773: 8-16.

45 Aberle H, Schwartz H, Kemler R: Cadherin-catenin complex: protein interactions and their implications for cadherin function. J Cell Biochem 1996; 61: 514-523.

46 Okamoto R, Irie K, Yamada A, Katata T, Fukuhara A, Takai Y: Recruitment of E-cadherin associated with alpha- and beta-catenins and p120ctn to the nectin-based cell-cell adhesion sites by the action of 12-0-tetradecanoylphorbol-13-acetate in MDCK cells. Genes Cells 2005; 10: 435-445.

47 Figueiredo J, Simoes-Correia J, Soderberg O, Suriano G, Seruca R: ADP-ribosylation factor 6 mediates E-cadherin recovery by chemical chaperones. PLoS One 2011; 6: e23188.

48 Bajpai S, Correia J, Feng $Y$ et al: \{alpha\}-Catenin mediates initial E-cadherindependent cell-cell recognition and subsequent bond strengthening. Proc Natl Acad Sci USA 2008; 105: 18331-18336.

49 Simoes-Correia J, Figueiredo J, Lopes R et al: E-cadherin destabilization accounts for the pathogenicity of missense mutations in hereditary diffuse gastric cancer. PLoS One 2012; 7: e33783.

50 Mateus AR, Simoes-Correia J, Figueiredo J et al: E-cadherin mutations and cell motility: a genotype-phenotype correlation. Exp Cell Res 2009; 315: 1393-1402. 